\title{
Antidepressants Reduce the Risk of Suicide among Elderly Depressed Patients
}

\author{
Yoram Barak*,', Ahikam Olmer' and Dov Aizenberg' \\ 'Abarbanel Mental Health Center and Geha Mental Health Center, Affiliated with the Sackler Faculty of Medicine, Tel-Aviv University, \\ Bat-Yam, Israel
}

\begin{abstract}
Treatment with selective serotonin reuptake inhibitors (SSRIs) may increase the risk of impulsive acts including suicide, while data from epidemiological studies suggest that the effect of SSRIs in the elderly may be beneficial. We aimed to evaluate the association between exposure to antidepressants and suicidality in a cohort of elderly patients suffering from major depressive disorder (MDD). This was a retrospective matched case-controlled evaluation over a I0-year period. All records of admissions of patients with MDD (ICD- I0) were assessed. The index group comprised all patients who had attempted suicide in the month prior to admission. The case-controlled group was the next admission of a patient suffering from MDD, matched for sex and age who had not attempted suicide in the month prior to admission. The index group during the 10-year period (1995-2004) consisted of 101 patients suffering from MDD who were hospitalized following a suicide attempt. Mean age for the group was $76.5 \pm 6.6$ years; there were 42 men and 59 women. The control group patients $(\mathrm{N}=10 \mathrm{I})$ were matched for age (mean $76.6 \pm 6.9$ years) and sex. The proportion of patients exposed to an antidepressant was significantly greater in the control group, than in the group of patients who had attempted suicide (58 vs $42 \%$, odds ratio 1.94 (95\% Cl: I.I-3.4), $p=0.019$ ). SSRIs were prescribed in $29 \%$ of patients in the control group vs $21 \%$ of patients in the index group ( $p=0.03$ ). It is of interest to note that concomitant prescription of benzodiazepines also conferred a protective effect. In conclusion, elderly depressed patients treated with antidepressants may be at reduced risk of attempting suicide. These findings need support from prospective randomized trials.
\end{abstract}

Neuropsychopharmacology (2006) 31, 178-181. doi:I0.1038/sj.npp. I 300863; published online 3 August 2005

Keywords: suicide; depression; elderly; SSRIs

\section{INTRODUCTION}

The most significant risk factors for attempted suicide include mood disorders and a history of suicide attempts. Suicide risk is assessed along a continuum from suicidal ideation alone to completed suicide. Although the incidence of suicide is low in the general population, it was among the leading causes of death in the Western world in 2000. Adolescents and the elderly are particularly at risk for suicide (Gaynes et al, 2004; Charney et al, 2003). Seniors aged 75 years and above have the highest suicide rates of all age groups in most industrialized countries (Waern et al, 2003).

Despite the availability of efficacious treatments, late-life depression remains improperly diagnosed and inadequately treated. Antidepressants are increasingly prescribed since the 1990s, yet pharmacotherapeutic treatment of depres-

*Correspondence: Professor Y Barak, Abarbanel Mental Health Center, I5 KKL Street, Bat-Yam, 59I00, Israel, Tel: 97235552738 , Fax: 9723 5552738, E-mail: mdybarak@netvision.net.il

Received 8 February 2005; revised 29 June 2005; accepted 5 July 2005 Online publication: 7 July 2005 at http://www.acnp.org/citations/ NPP070705050082/default.pdf sion is still inadequate due to insufficient dosing and short period of treatment (Fischer et al, 2003; Salzman et al, 2002).

The estimated population attributable risk for mood disorder in elderly suicide is $74 \%$; that is, if mood disorders were eliminated from the population, $74 \%$ of suicides would be prevented in the elderly (Beautrais, 2002). 'Elimination' of mood disorders is possible not only by treatment of existing cases but also by primary and secondary prevention (O'Connell et al, 2004). In recent years, attempts have been made to prevent suicide in the community through various interventions in primary care, with particular emphasis on elderly subjects since they are at high risk. One of the leading trials, known as Prevention of Suicide in Primary Care Elderly: Collaborative Trial (PROSPECT), recruited 598 patients from 20 primary care practices in the USA. The PROSPECT algorithm recommended a first-line trial of a selective serotonin reuptake inhibitor (SSRI), as this reflects the reality of primary care. The intervention's effectiveness in reducing suicidal ideation reinforces its role as a prevention strategy to reduce risk factors for suicide in late life (Bruce et al, 2004). The demonstrated effect in the PROSPECT trial was already predicted by Freemantle et al (1994) concerning the population of England, as follows: 
'The potential number of lives which may be saved from a switch to the routine first line use of SSRIs is between 300 and 450 each year'. Despite the enthusiasm shown by several research groups for the use of SSRIs as potently decreasing the risk of suicide, others have reported increased impulsivity and increased rates of suicide associated with exposure to SSRIs (Teicher et al, 1993; Donovan et al, 1999; Healy, 2003). In a recent review of several meta-analyses, Healy and Whitaker (2003) emphasized the need to identify the characteristics of patients who may be most at risk. Thus, the present study was designed to assess the effects of exposure to antidepressants in elderly patients suffering from major depression-possibly the group of patients at the highest risk of suicide.

\section{METHOD}

There are no private emergency psychiatric facilities in Israel, and all acute psychiatric hospitalizations are regional and reported to the Ministry of Health.

The Abarbanel Mental Health Center, Bat-Yam, Israel, serves a catchment area of approximately 680000 subjects. The center is affiliated to the Sackler School of Medicine, Tel-Aviv University. There are 470 in-patient beds and 60 day patients, as well as a large outpatient clinic. This is a tertiary psychiatric facility in contact with both secondary specialist clinics and primary care providers. The services for the elderly include two in-patient departments, an outpatient clinic, and outreach consultancy services for nursing homes within the catchment area.

The present study is a retrospective analysis of all records of elderly patients suffering from major affective disorder (unipolar depression, MDD) admitted to our center over a period of 10 years. The center's Internal Review Board (IRB) approved this study.

The Israeli Ministry of Health requires that there be a record of suicide attempt in the 4 weeks preceding the hospitalization for every admission to a psychiatric ward. The records are transferred to the Ministry weekly and a copy is kept at every site. During the period January 1995 and December 2004 there were 852 admissions to our center of elderly patients who had attempted suicide prior to hospitalization. This data set $(N=852)$ was queried for all elderly patients fulfilling the following inclusion criteria: (1) ICD-10 diagnosis of depressive episode or recurrent depressive episode (F32 and F33 codes; all subtypes) and (2) availability of treatment charts.

Patients who had fulfilled these criteria were defined as the index group (ie, elderly major depressive disorder (MDD) patients who had attempted suicide within 1 month prior to admission). The case-controlled group comprised the next admission of an elderly patient suffering from MDD, with available treatment chart, matched for sex and age (within 1 year) who had not attempted suicide within 1 month prior to hospitalization.

Computerized index and control patient files were reviewed and the following data extracted: age, sex, diagnosis subtype, suicide attempt prior to admission (index patients), treatment with psychotropic medication, manner of suicide attempt (index patients), and history of prior suicide attempts.

\section{Statistical Analysis}

Data were analyzed using the nonparametric sign-rank test for paired groups. This method was employed due to uneven distribution of within-group differences. The $t$-test and a nonparametric test were undertaken to test for differences between the evaluations for continuous variables. Examination of differences between the categorical parameters was based on the McNemar test for dependent (matched) populations. All tests applied were two tailed, and a $p$-value of $5 \%$ or less was considered statistically significant.

The data were analyzed using the Statistical Analysis System software, SAS Institute (1990).

\section{RESULTS}

During the study period, 205 elderly MDD were hospitalized at the Abarbanel Mental Health Center psycho-geriatric division following a suicide attempt. Of these, $101 \mathrm{MDD}$ patients fulfilled all inclusion criteria and are the index group of the present analysis. The 104 discarded files were missing data as to the details and manner of the suicide attempt $(N=82)$ and treatment charts $(N=22)$.

The clinical characteristics of cases and controls are presented in Table 1.

The index group thus analyzed had a majority of women (59); mean age for the index group was $76.5 \pm 6.6$ years and the depressive episode was a recurrent one (F33 code; ICD$10)$ in $66 \%$ of cases. The control group also consisted of 101 patients matched for age (mean $76.6 \pm 6.9$ years) and sex ( 42 men and 59 women). In $72 \%$ of control group patients, the

Table I Clinical Characteristics of Cases and Controls

\begin{tabular}{lccc}
\hline Variable & $\begin{array}{c}\text { Cases } \\
(\mathbf{N}=\mathbf{I 0 I})\end{array}$ & $\begin{array}{c}\text { Controls } \\
(\mathbf{N}=\mathbf{I 0 I})\end{array}$ & p-value (df) \\
\hline Age (years) & $\begin{array}{c}76.5 \\
( \pm 6.6)\end{array}$ & $\begin{array}{c}76.6 \\
( \pm 6.9)\end{array}$ & $\mathrm{NS}$ \\
& & & \\
Gender & $59 \mathrm{~F}$ & $59 \mathrm{~F}$ & $\mathrm{NS}$ \\
& $42 \mathrm{M}$ & $42 \mathrm{M}$ & $(\mathrm{df}=58,4 \mathrm{I}$, respectively)
\end{tabular}

$\begin{array}{lllc}\text { Diagnosis } & & & \\ \text { F32 (single episode) } & 34 & 28 & \text { NS } \\ \text { F33 (recurrent) } & 67 & 73 & (\mathrm{df}=27,65, \text { respectively })\end{array}$

$\begin{array}{lrrr}\text { Psychotropic treatment (\%) } & & & \\ \text { Antidepressants (A) } & 42 & 58 & 0.02(\mathrm{df}=4 \mathrm{I}) \\ \text { Benzodiazepines (B) } & 14 & 28 & 0.026(\mathrm{df}=13) \\ \text { Antipsychotics } & 18 & 14 & \mathrm{NS}(\mathrm{df}=13) \\ \text { Mood stabilizers } & 3 & 3 & \mathrm{NS}(\mathrm{df}=2) \\ \text { A+B } & 86 & 93 & \mathrm{NS}(\mathrm{df}=85)\end{array}$

Past suicide attempt $\quad 41 \quad 15 \quad 0.003(\mathrm{df}=14)$

$\mathrm{F}=$ female, $\mathrm{M}=$ male

$F 32, F 33=I C D-10$ codes.

$A+B=$ combined treatment with both antidepressants and benzodiazepines. 
depressive episode was recurrent. Age, sex, and depression subtype did not differ between groups ( $t$ value $=0.01$, $\mathrm{df}=201, p=0.93, \chi^{2}=0.0036, \mathrm{df}=1, p=1.0, \chi^{2}=0.0928$, $\mathrm{df}=1, p=0.92$, respectively).

The exposure to antidepressant medications was significantly different between groups. While $42 \%$ of elderly MDD patients who had attempted suicide prior to hospitalization received antidepressant treatment (index group), 58\% of patients in the control group were exposed to antidepressant treatment (a 38\% higher rate). This increased rate of exposure was statistically significant, $\chi^{2}=5.4, \mathrm{df}=1, p=0.02$. Exposure to antidepressants conferred a protective effect with an odds ratio, $\mathrm{OR}=1.94,95 \%$ confidence interval, $\mathrm{CI}=1.1-3.4$.

The various antidepressant drugs groups were distributed differently between the index and control groups. The most common antidepressants in both groups were the SSRIs, followed by the tricyclics, and then the SNRIs. However, the rates of prescription differed between groups with the highest exposure to SSRIs in the control group 29\% of all patients $v s 21 \%$ in the index group, $19 \%$ tricyclics in both groups, SNRIs in $7 \%$ of the control group vs $2 \%$ of the index group, and RIMA in $3 \%$ of the control group $v s 0 \%$ of the index group $\left(\chi^{2}=10.6, \mathrm{df}=4, p=0.031\right)$.

Patients had been treated with additional psychotropic compounds. The most frequently prescribed group of drugs was benzodiazepines (28\% control group vs $14 \%$ of the index group), antipsychotics (14 vs 18\%), and mood stabilizers (3vs 3\%). The increased rate of benzodiazepines prescription among the control group subjects was statistically significant, $\chi^{2}=12.7, \mathrm{df}=5, p=0.026$. It is important to note that benzodiazepines were coprescribed with an antidepressant in the majority of patients in both the index and control groups (86 and 93\%, respectively).

The majority of patients who had attempted suicide did so by drug overdose ingestion (54\%) followed by wrist cutting $(16 \%)$ or jumping from heights (11\%). In an analysis of risk, it was demonstrated that patients with a history of previous suicide attempt were significantly overrepresented in the index group; 41 vs $15 \%$ in the control group, $p=0.003$. The risk ratio for attempted suicide in a patient with a previous attempt was OR $=3.69(95 \%$ $\left.\mathrm{CI}=1.9-7.2, \chi^{2}=15.6, \mathrm{df}=1, p=0.0001\right)$.

\section{DISCUSSION}

The present study indicates that elderly patients treated with antidepressants, mainly SSRIs, are significantly less likely to have attempted to commit suicide within the month previous to hospitalization for MDD than a cohort matched for age and sex. Recent discussions by researchers, regulators, and consumers have presented arguments in favor of a suicidogenic potential of the SSRIs. This effect is thought to be more pronounced in children and adolescents. However, reviewing epidemiological data of Western populations does not support the hypothesis that antidepressants or, more specifically, SSRIs cause increased suicidality in elderly patients with depression (Lapierre, 2003). Two important examples are worth mentioning. First, antidepressant prescribing in Northern Ireland has increased over five-fold in the decade 1989-1999. In younger adults there was no association between antide- pressant prescribing and suicide. For the older group increased antidepressant prescribing was associated with a reduction in suicide rate over the 10 years of the study (Kelly et al, 2003). Second, Hall et al (2003) examined the association between trends in antidepressant prescribing and suicide rates in Australia for 1991-2000. Again, while overall national rates of suicide did not fall significantly, incidence decreased in older men and women. These authors have suggested that the increase in antidepressant prescribing may be a proxy marker for improved overall management of depression and, thus, increased prescribing of SSRIs in general practice may have produced a quantifiable benefit (Hall et al, 2003).

To the best of our knowledge, the present study is the first to directly test the association between exposure to antidepressants and suicide attempts among elderly MDD patients in a case-controlled design. Our findings are consistent with data from epidemiological analyses that describe a direct association between increased SSRIs prescribing and decreased suicide rates in the elderly. In the present study 'real-life' effect was analyzed, in contrast to epidemiological studies, in which population trends are explored.

The present study has several methodological and datarelated limitations. Compliance, which may affect suicidality, was not accounted for. The pharmacological data had limitations such as that treatments were not standardized, data were collected retrospectively, and reliable information on doses and treatment duration were unavailable. Previous suicide attempts are a known risk factor for late life suicide. These were not controlled for in the present study and thus may have added to group differences. A significant weakness of this study is the low rate of depressed patients among subjects who had attempted suicide and the difficulty of extracting complete data in half of these cases. This had limited our ability to match the control group for clinical characteristics such as disease severity. In addition, the admission of elderly patients to our tertiary center not following a suicide attempt may have been due to different depression severity, physical frailty or compliance problems. These inter-group differences need be taken into account and temper the study's conclusions. On the other hand, the study's strengths are the matching of index patients and control patients as to age and gender, the relatively large sample size, and the compulsory reporting of suicide attempts demanded by the health authorities.

Recently, using variation across countries over time in SSRI sales and suicide Ludwig and Marcotte (2005) suggested that expanding access to SSRIs for adults may be a cost-effective way to save lives. However, they caution that analyzed randomized trials are not fully informative on this question because of small sample sizes and other limitations. The findings of the present study are of similar trend. In conclusion, we suggest that for elderly patients suffering from depression, treatment with SSRIs may confer a protective effect from suicidality. Further prospective studies are needed to confirm our findings.

\section{ACKNOWLEDGEMENTS}

This study was supported by an unrestricted research grant from $\mathrm{H}$ Lundbeck A/S. 


\section{REFERENCES}

Bruce ML, Ten Have TR, Reynolds III CF, Katz II, Schulberg HC, Mulsant BH et al (2004). Reducing suicidal ideation and depressive symptoms in depressed older primary care patients: a randomized controlled trial. JAMA 291: 1081-1091.

Beautrais AL (2002). A case control study of suicide and attempted suicide in older adults. Suicide Life Threat Behav 32: 1-9.

Charney DS, Reynolds III CF, Lewis L, Lebowitz BD, Sunderland T, Alexopoulos GS et al (2003). Depression and Bipolar Support Alliance consensus statement on the unmet needs in diagnosis and treatment of mood disorders in late life. Arch Gen Psychiatry 60: 664-672.

Donovan S, Kelleher MJ, Lambourn J, Foster R (1999). The occurrence of suicide following the prescription of antidepressant drugs. Arch Suicide Res 5: 181-192.

Fischer LC, Wei F, Solberg LI, Rush WA, Heinrich RL (2003). Treatment of elderly and other adult patients for depression in primary care. J Am Geriatr Soc 51: 1554-1562.

Freemantle N, House A, Song F, Mason JM, Sheldon TA (1994). Prescribing selective serotonin reuptake inhibitors as strategy for prevention of suicide. BMJ 309: 249-253.

Gaynes BN, West SL, Ford CA, Frame P, Klein J, Lohr KN (2004). Screening for suicide risk in adults: a summary of the evidence for the US Preventive Services Task Force. Ann Intern Med 140: 822-835.
Hall WD, Mant A, Mitchell PB, Rendle VA, Hickie IB, McManus P (2003). Association between antidepressant prescribing and suicide in Australia, 1991-2000: trend analysis. BMJ 326: 1008-1013.

Healy D (2003). Lines of evidence on the risks of suicide with selective serotonin reuptake inhibitors. Psychother Psychosom 72: 71-79.

Healy D, Whitaker C (2003). Antidepressants and suicide: riskbenefit conundrums. J Psychiatry Neurosci 28: 331-337.

Kelly CB, Ansari T, Rafferty T, Stevenson M (2003). Antidepressant prescribing and suicide rate in Northern Ireland. Eur Psychiatry 18: 325-328.

Lapierre YD (2003). Suicidality with selective serotonin reuptake inhibitors: valid claim? J Psychiatry Neurosci 28: 340-347.

Ludwig J, Marcotte DE (2005). Anti-depressants, suicide, and drug regulation. J Policy Anal Manage 24: 249-272.

O'Connell H, Chin AV, Cunningham C, Lawlor BA (2004). Recent developments: suicide in older people. BMJ 16: 895-899.

Salzman C, Wong E, Wright BC (2002). Drug and ECT treatment of depression in the elderly, 1996-2001: a Literature Review. Biol Psychiatry 52: 265-284.

SAS/STAT (1990). Users' Guide Version 6, 4th edn, Vols 1, 2. SAS Institute: Cary, NC.

Teicher MH, Glod CA, Cole JO (1993). Antidepressant drugs and the emergence of suicidal tendencies. Drug Saf 8: 186-212.

Waern M, Rubenowitz E, Wilhelmson K (2003). Predictors of suicide in the old elderly. Gerontology 49: 328-334. 